\title{
Direct ocean surface velocity measurements from space: Improved quantitative interpretation of Envisat ASAR observations
}

\author{
J.A.Johannessen ${ }^{1,2, *}$, B. Chapron ${ }^{3}$, F. Collard ${ }^{4}$, V. Kudryavtsev ${ }^{5,6,1}$, A. Mouche ${ }^{4}$, D. Akimov ${ }^{5}$, and \\ K.-F. Dagestad ${ }^{1}$
}

${ }^{1}$ Nansen Environmental and Remote Sensing Center, Bergen, Norway Thormoehlensgate 47, N-5006, Bergen Norway

${ }^{2}$ Geophysical Institute, University of Bergen, Norway

${ }^{3}$ Institute Francais de Recherche pour I'Exploitation de la Mer, Plouzané, France

${ }^{4}$ CLS - Direction of Radar Applications, Plouzané, France

${ }^{5}$ Nansen International Environmental and Remote Sensing Center, St. Petersburg, Russia

${ }^{6}$ Marine Hydrophysical Institute, Sebastopol, Ukraine

*: Corresponding author : J.A.Johannessen, email address : johnny.johannessen@nersc.no

\begin{abstract}
:
Previous analysis of Advanced Synthetic Aperture Radar (ASAR) signals collected by ESA's Envisat has demonstrated a very valuable source of high-resolution information, namely, the line-of-sight velocity of the moving ocean surface. This velocity is estimated from a Doppler frequency shift, consistently extracted within the ASAR scenes. The Doppler shift results from the combined action of near surface wind on shorter waves, longer wave motion, wave breaking and surface current. Both kinematic and dynamic properties of the moving ocean surface roughness can therefore be derived from the ASAR observations. The observations are compared to simulations using a radar imaging model extended to include a Doppler shift module. The results are promising. Comparisons to coincident altimetry data suggest that regular account of this combined information would advance the use of SAR in quantitative studies of ocean currents.
\end{abstract}




\section{Introduction}

SAR measurements offer a potential to map current divergence and convergence zones, where distinct upper layer dynamics, changes in wave properties and coupling to biogeochemical processes occur. Kudryavtsev et al. (2005) and Johannessen et al. (2005) proposed a practical radar imaging model (RIM) to advance the quantitative interpretation of high resolution radar measurements of surface current features. This model explicitly builds on a particular decomposition of the sea surface into a background of regular small wave slopes and heights covering most of the surface, and fewer isolated very rough patches of intermittent steep waves with large curvature and breaking waves.

Using the SAR high resolution processing principle, Chapron et al. (2005) pioneered the method to retrieve the line-of-sight radar-detected ocean surface roughness velocity from single antenna satellite SAR measurements. Regular access to Doppler shift measurements from ASAR Wave Mode (WM) and Wide Swath Mode (WSM) images has been possible only since mid 2007, providing an increasing data set of both kinematic and dynamic properties of the radar-detected moving ocean surface roughness. This is demonstrated in Figure 1 where the influence of the greater Agulhas Current is visible in the line-of-sight (ground range) Doppler velocity captured by the ASAR sensor.

The single-antenna Doppler shift anomalies are obtained by subtracting the predicted from the measured Doppler centroids. The method works best for images with quasi-uniform radar cross-section at moderate to higher winds, predominantly used in this study, and yield estimates with a resolution (azimuth, range) of about $10 \mathrm{~km}$ by $6 \mathrm{~km}$ for WM imagettes and about $8 \mathrm{~km}$ by $4 \mathrm{~km}$ for WSM images with $30 \%$ overlap in azimuth. For WSM products, prior to geophysical interpretations, corrections are applied to compensate along-track large cross section variations and biases are further removed using land surface references. For WM products, biases are removed for each orbit. The resulting Doppler anomalies are then obtained with an RMS error up to $5 \mathrm{~Hz}$, equivalent to respectively $0.35 \mathrm{~m} / \mathrm{s}$ and $0.21 \mathrm{~m} / \mathrm{s}$ in range directed surface Doppler velocity at $23^{\circ}$ and $33^{\circ}$ incidence angles.

The Agulhas Current regime has been described as one of the strongest western boundary currents (up to $2 \mathrm{~m} / \mathrm{s}$ ) in the world's oceans. The estimated radial Doppler velocity reaching up towards $2 \mathrm{~m} / \mathrm{s}$ (Figure 1) appears to map the expression of this current. Passing the retroflection region centered at $16^{\circ} \mathrm{E}$, the Agulhas return current meanders eastward back into the South Indian Ocean between $38^{\circ}-40^{\circ} \mathrm{S}$. This reversal of the mean flow translates into opposite sign radial surface Doppler velocities reaching up to $1.5 \mathrm{~m} / \mathrm{s}$ (Figure 1). The persistent manifestation of these Doppler velocity signatures and the apparent agreement to the location of the core geostrophic current derived from weakly map of altimetry are certainly striking.

Although the Doppler velocity is not a direct surface current measurement, it inevitably suggests that the use of Doppler observations can help to derive new and innovative estimates of the mesoscale dynamics. To reach consistent quantitative results, a semiempirical model is highly preferable to guide quantitative interpretation based on both surface roughness variation and Doppler anomaly analyses. In this paper, the RIM model extended with the Doppler module is used to predict the expected Doppler shift. The approach is described in section 2. Model results are compared to Envisat C-band ASAR WM and WSM Doppler frequency shift measurements in section 3, followed by a summary in section 4 .

\section{Approach}

The RIM builds on a two-scale asymptotic decomposition and derivation of the Doppler velocity is straightforward (Appendix B in Chapron et al., 2005). Accordingly, a sea surface normalized radar cross-section, (NRCS, $\sigma_{0}$ ), is defined locally. It is then modulated and experiences local vertical and horizontal movements due to longer surface waves. Over an ocean imaged scene, the Doppler frequency $f_{D}$ becomes a mean quantity, 


$$
\frac{\pi f_{D}}{k_{R}}=-\frac{\overline{(u \sin \theta-w \cos \theta) \sigma_{0}(\theta+\Delta \theta)}}{\overline{\sigma_{0}(\theta+\Delta \theta)}}
$$

Here $k_{R}$ is the radar wavenumber, $u$ and $w$ are the horizontal and vertical velocities of the scattering facets, and $\Delta \theta$ is the modification of the incidence angle $\theta$ due to the local tilt induced by the longer waves. This two-scale assumption helps to consider the NRCS variations caused by both the change of the local surface tilt $(\Delta \theta)$ and the hydrodynamic modulation $\left(\tilde{\sigma}_{0}^{h}\right)$ of the scattering facets, as $\tilde{\sigma}_{0}=\Delta \theta \frac{\partial \sigma_{0}}{\partial \theta}+\tilde{\sigma}_{0}^{h}$, where $\Delta \theta=-\left(\cos \varphi_{R} \cdot \varsigma_{x}+\sin \varphi_{R} \cdot \varsigma_{y}\right), \varphi_{R}$ is the radar look direction, and $\varsigma_{x}, \varsigma_{y}$ are the local components of the sea surface slope. We ignore effects of surface tilt out of the incidence plane. To the second order in steepness, the radial velocity $V_{D}$ of the target (assumed positive if directed away from the radar) writes

$$
V_{D}=-\pi f_{D} / k_{R} \sin \theta=\bar{c}_{f}+u_{S}+c_{f}^{T H}
$$

where $\bar{c}_{f}$ is the mean velocity of the scattering facets, and $u_{S}$ is the radial surface current velocity. As hypothesized, facets travel along large-scale surface waves composed from a wide spectrum of waves with $k<k_{L}$ (where $k_{L}$ is a spectral cutoff linked to the scale of the facets), and $C_{f}^{T H}$, is the contribution due to tilting and hydrodynamic modulation of the facets. $c_{f}^{T H}$ can be expressed as:

$$
c_{f}^{T H}=\int_{k<k_{L}}\left[\left(-\cot \theta \cdot M_{f}^{t}+M_{1 f}^{h}\right) \cos \left(\varphi_{R}-\varphi\right)+\cot \theta \cdot M_{2 f}^{h}\right] \cdot c k^{-2} B(\mathbf{k}) d \mathbf{k}
$$

where $M_{f}^{t}=\partial \ln \left(\sigma_{0}\right) / \partial \theta$ is the tilt modulation transfer function (MTF), $M_{f}^{h}=M_{1 f}^{h}+i \cdot M_{2 f}^{h}$ is the hydrodynamic MTF (real $M_{1 f}^{h}$ and imaginary $M_{2 f}^{h}$ part describes correlation of a scattering facets modulations with elevations and slopes of the modulating waves), $B(\boldsymbol{k})$ is the 2D saturation spectrum, and $\varphi$ is the direction of $\boldsymbol{k}$. The two first terms in (3) provide changes of sign in $c_{f}^{T H}$ if $\varphi_{R}$ turns from down- to up-wind, while effect of facets-slopes correlation (third term in (3) is not dependent on $\varphi_{R}$ and provides up- and down-wind asymmetry in $V_{D}$.

RIM assumes the NRCS to be represented by the sum: $\sigma_{0}^{p}=\sigma_{0 R}^{p}(1-q)+\sigma_{0 b} q$ where $\sigma_{0 R}^{p}$ and $\sigma_{0 b}$ are the NRCS of the regular surface (at $\mathrm{p}=\mathrm{vv}$ - or hh-polarization) and the non regular surface of breakers covering a fraction $q$ of the sea surface. $\sigma_{0 R}^{p}$ follows a composite model leading to the sum of two terms, i.e. the so-called two-scale Bragg and quasi-specular contributions, $\sigma_{0 R}^{p}=\sigma_{s p}+\sigma_{b r}^{p}$. The partial contributions to $\sigma_{0}^{P}$ become $P_{b r}^{p}=(1-q) \sigma_{b r}^{p} / \sigma_{0}^{p}, P_{s p}^{p}=(1-q) \sigma_{s p} / \sigma_{0}^{p}$, and $P_{w b}^{p}=q \sigma_{0 b} / \sigma_{0}^{p}$. As a key aspect, the RIM polarization ratio becomes controlled by the non-Bragg scalar scattering contribution. The RIM predictions are in good agreement with experimental data (e.g. by Mouche et al., 2006). 
The systematic and significant deviation between a standard composite-Bragg scattering model prediction and observations proved that the scalar term plays a crucial role, comparable to the sea surface curvature effect in advanced scattering model (e.g., Mouche et al., 2007a, 2007b). The radial Doppler velocity thus becomes:

$V_{D}=u_{s}+\sum P_{j}^{P}\left(\bar{c}_{j}+c_{j}^{T H}\right)$

with the subscript $\mathrm{j}$ representing Bragg waves (br), specular mirror points (sp) and breakers $(w b)$. For the Bragg-facets the spectral cutoff wavenumber $k_{L b r}$ is defined as $k_{L b r}=d k_{R}$ (with $d=1 / 4$ ), while the range of longer waves modulating the breaker-facets is limited to $k<k_{L w b}$ $=d k_{w b}=d k_{R} / 10$. For specular mirror points the dominant modulating waves $k_{L s p}$ are assumed to be equal to the peak wavenumber in the wind wave spectrum. Explicit expressions for the hydrodynamic modulations of each of the types of scattering facet can be found in Kudryavtsev et al. (2003b).

The mean line-of-sight velocity of the scattering facets $\bar{C}_{j}$ in eq. (4) is represented as a sum of the phase speed of the Bragg waves $\left(\bar{C}_{b r}\right)$, advection speed of "mirror points" $\left(\bar{C}_{s p}\right)$ and speed of breakers $\left(\bar{C}_{w b}\right)$. The advection speed of the "mirror points" is expressed following Longuet-Higgins (1957)

$$
\bar{C}_{s p}=\cos \varphi_{R} \int_{k<d \cdot k_{R}} \cos \varphi c k^{-2} B(\mathbf{k}) d \mathbf{k} / s_{u p}^{2}+\sin \varphi_{R} \int_{k<d \cdot k_{R}} \sin \varphi c k^{-2} B(\mathbf{k}) d \mathbf{k} / s_{c r}^{2}
$$

where up- and cross-wind mean squared slopes of the large-scale surface $\left(s_{u p}^{2}\right.$ and $s_{c r}^{2}$ ) are defined as $\left[s_{u p}^{2}, s_{c r}^{2}\right]=\int_{k<k_{L b r}}\left[\cos ^{2} \varphi, \sin ^{2} \varphi\right] \cdot k^{-2} B_{w}(\mathbf{k}) d \mathbf{k}$.

The breaker-facet velocity $\mathbf{c}$ is scale dependent and is described in terms of $\Lambda(\mathbf{c}) d \mathbf{c}$ that defines the length of wave breaking fronts per unit area with velocities ranging from $\mathbf{C}$ to $\mathbf{C}+d \mathbf{c}$ (Phillips, 1985). The quantity $k^{-1} \Lambda(\mathbf{c}) d \mathbf{c}$ is proportional to the fraction of the enhanced roughness area, and

$$
\bar{C}_{w b}=\int_{k<k_{R} / 10} \cos \left(\varphi-\varphi_{R}\right) c k^{-1} \Lambda(\mathbf{c}) d \mathbf{c} / \int_{k<k_{R} / 10} k^{-1} \Lambda(\mathbf{c}) d \mathbf{c}
$$

Assuming that the energy losses are proportional to the energy input from the wind, the spectral distribution of the breaking fronts in eq. (6) can be replaced by $\Lambda(\mathbf{c}) d \mathbf{c} \propto k^{-1} \beta B(\mathbf{k}) d \mathbf{k}$ where $\beta \propto\left(u_{*} / c\right)^{2}$ is the wind wave growth rate.

\section{Model results and comparisons}

Results of the extended RIM - Doppler model (hereinafter DopRIM) are presented and compared to Doppler anomalies obtained from the global Envisat ASAR WM data. Following eq. (4), the partitioning of the scattering contributions plays an essential role to quantify the individual contributions to the total Doppler velocity. Each weight is wind speed and direction dependent, as well as incidence angle and polarization dependent. The specular point 
velocity always dominates $V_{D}$ at low incidence angle. With increasing incidence angles, this part of the Doppler velocity becomes negligible. At moderate incidence angles, the simulation of the total Doppler velocity predicts values that are about $35 \%$ of the wind speed. This is significantly larger than expected from the phase speed of the Bragg waves and the wind induced surface drift (about 3\% of wind speed). The two-scale decomposition with tilting and hydrodynamic effects explains this difference. More specifically, at moderate incidence angles, the composite-Bragg facet velocity is larger for $\mathrm{HH}$ than for $\mathrm{VV}$ polarization. This is anticipated from the larger tilting effects at $\mathrm{HH}$ than at $\mathrm{VV}$. The composite non-Bragg facet velocity has a relatively small weight for $\mathrm{VV}$. On the other hand, for $\mathrm{HH}$ polarization, following the RIM prescribed reduction of the polarization ratio, the composite-Bragg and singular scattering contributions become equal. Consequently, at moderate to large incidence angles, the breaking contribution cannot be neglected, and for $\mathrm{HH}$, it eventually dominates $V_{D}$ at very large angles.

Using WM data the observed and simulated wind dependence of C-band Doppler shift for VV and $\mathrm{HH}$ polarization are plotted for the $23^{\circ}$ and $33^{\circ}$ incidence angles in Figure 2. Overall the simulated Doppler frequency shifts display a functional relationship versus wind speed in good agreement with the observations, in particular up to a wind speed of $+/-15 \mathrm{~m} / \mathrm{s}$, with a mean difference gradually increasing from about $2 \mathrm{~Hz}$ for $\mathrm{VV}$ at $23^{\circ}$ to $5 \mathrm{~Hz}$ for $\mathrm{HH}$ at $33^{\circ}$. The observed Doppler anomaly differences between $\mathrm{HH}$ and $\mathrm{VV}$ are generally small, and assumingly related to the relatively weak NRCS polarization ratio measured at C-band. Under the RIM decomposition, the scalar contributions must play a significant role. Further investigations should therefore be directed to explain both the weak polarization ratio and the small Doppler anomaly differences. As the number of ASAR Doppler frequency shift observations is growing, this will become feasible.

In revisiting the expressions of the Agulhas Current captured in the WSM Doppler velocity time series further quantitative analyses is now possible taking into account the relationship presented above. The core position of the maximum surface geostrophic current derived from the 7-day (15-22 September) composite altimeter map (Figure 3, left) is superimposed on the full Doppler velocity map derived from ASAR (Figure 3, middle). The mean location and flow direction of the southern part of the Agulhas Current and the evidence of the Agulhas return current agrees very well. It is also worth noting that although the return current orientation is rotated away from range direction, its radial component is clearly manifested. Comparison of range directed velocities along the red-stippled line (Figure 3, middle) reveals, however, distinct differences in magnitude (Figure 3, right). In particular at the core of the Agulhas Current, where the maximum surface geostrophic current is only about $0.7 \mathrm{~m} / \mathrm{s}$ compared to the Doppler velocity that reaches nearly $2 \mathrm{~m} / \mathrm{s}$. This latter speed is also reported from surface drifters trapped in the current (www.meds-sdmm.dfo-mpo.gc.ca). Effect of topographic steering plus time-space averaging of the altimeter data superimposed on a smooth $200 \mathrm{~km}$ resolution mean dynamic topography applied in the construction of the weekly mean surface geostrophic map are assumed to explain some of this underestimation.

By invoking the easterly, radial directed $4-10 \mathrm{~m} / \mathrm{s}$ ECMWF wind speed into DopRIM the simulated wind contribution to the Doppler velocity is found to be rather smooth with a speed varying from 0.5 to $0.75 \mathrm{~m} / \mathrm{s}$ in the ASAR look direction (Figure 3c). The $100 \mathrm{~km}$ wide and opposite directed Doppler speed reaching nearly $2 \mathrm{~m} / \mathrm{s}$ with an estimated accuracy of about $0.2 \mathrm{~m} / \mathrm{s}$ and with a maximum shear of about $10^{-4} \mathrm{~s}^{-1}$ is therefore predominantly reflecting the influence of the Agulhas Current on the Doppler velocity measurement. The same is also valid for the $1.5 \mathrm{~m} / \mathrm{s}$ Doppler speed of the Agulhas Return Current. This suggests that it is possible to derive quantitative information of these intense surface currents from the radial Doppler velocity. Using this method in combination with surface drifters and altimeter derived surface geostrophic current could consequently strengthen the ability to study surface current dynamics. 


\section{Summary}

In this study, the DopRIM has been defined and used to consistently examine and remove the dependence of the Doppler velocity on radar parameters and sea surface radar scatter moving elements. In particular the impact of intermittent steep events with large curvature and existence of breaking waves is incorporated. This effect was mainly introduced to simulate the observed weak polarization ratio of the sea surface backscatter and reduce the differences between VV and $\mathrm{HH}$ Doppler shifts. Tilting and hydrodynamic effects are taken into account, and the relative velocity contribution associated with non-Bragg roughness elements becomes rapidly preponderant for $\mathrm{HH}$ measurements. This has also recently been emphasized by Mouche et al. (2008) using an advanced scattering model.

DopRIM helps to refine the distinct relationship between range-directed Doppler velocity and wind speed in agreement with the newly available ASAR WM observations for wind speeds in the range of $+/$ - $15 \mathrm{~m} / \mathrm{s}$. Moreover, quantitative assessment of the Doppler shifts encountered in WSM observations of the intense and persistent Agulhas Current with variable dominance of shear, convergence and divergence zones then yields promising results. The greater Agulhas Current makes an ideal natural laboratory for these WSM Doppler shift measurements, as will Doppler shift measurements in the presence of mesoscale eddies. At a spatial (azimuth - range) resolution of $8 \mathrm{~km}$ by $4 \mathrm{~km}$ a maximum speed near $2 \mathrm{~m} / \mathrm{s}$ was obtained in the core of the Agulhas Current with an estimated error of $0.2 \mathrm{~m} / \mathrm{s}$ in Doppler velocity at $40^{\circ}$ incidence angle. In contrast the weekly mean surface geostrophic current derived from altimetry reached only $0.6-0.7 \mathrm{~m} / \mathrm{s}$.

Advancing the quantitative estimation of surface current dynamics also implies new possibilities to explore the coupling to biogeochemical processes that often occurs through ageostrophic processes along fronts and within eddies, usually well traced by local radar cross-section intensity contrasts.

In summary, the results are considered promising for strengthening the use of SAR in quantitative studies of the ocean currents. Combined with surface drifters and altimeterderived surface geostrophic current, monitoring of the dynamics of intense current regimes may be advanced. Furthermore, as persistent feedback exists between the near surface wind, sea surface temperature and surface current in frontal regions, these new consistent kinematic and dynamic ASAR-based observations will also improve studies of air-sea interaction processes in vicinity of strong current regimes. The accuracies of the Doppler shift and - velocity need careful assessment, in particular to quantify instrumental and geophysical contributions to the error budget. Such quantification will be very challenging. A dedicated validation campaign with adequate sensors is therefore highly needed, preferably in an intense and broad current regime, such as the Agulhas Current, with its optimum current flow direction versus the radar look direction. This would be very timely in view of Sentinel-1, which is planned for launch in 2012 to ensure continuity of C-band SAR data in support to Global Monitoring for Environment and Security (GMES).

\section{Acknowledgements}

This work was supported by the Norwegian Space Centre and the European Space Agency (ESA) through the Prodex Arrangement with contract no. 90266, and by the Research Council of Norway, project number $177441 / \mathrm{V} 30$. Support by ESA through the study contract no. 18709/05/I-LG, Service Hydrographique et Océanographique de la Marine (SHOM) through contract 05.87.028.00.470.29.25, and INTAS Association through the INTAS-06-10000259264 project are also acknowledged. We are also grateful for the altimeter data obtained from www.aviso.oceanobs.com , AVISO-Centre National d'Etudes des Spatiales (CNES). 


\section{References}

Chapron, B., F. Collard, and F. Ardhum, (2005), Direct measurements of ocean surface velocity from space: Interpretation and validation, Journal of Geophysical Research, 110, C07008.

Johannessen, J.A., V.Kudryavtsev, D.Akimov, T. Eldevik, N. Winther, and B. Chapron, (2005), On Radar Imaging of Current Features; Part 2: Mesoscale Eddy and Current Front detection. Journal of Geophysical Research, Vol. 110, C07017.

Kudryavtsev, V., D. Hauser, G. Caudal, and B. Chapron, (2003a), A semi-empirical model of the normalized radar cross-section of the sea surface. Part 1: The background model, Journal of Geophysical Research, 108(C3), 8054, doi:10.1029/2001JC001003.

Kudryavtsev, V., D. Hauser, G. Caudal, and B. Chapron, (2003b), A semi-empirical model of the normalized radar cross-section of the sea surface. Part 2: Radar modulation transfer function, Journal of Geophysical Research, 108(C3), 8055, doi:10.1029/2001JC001004.

Kudryavtsev, V., D. Akimov, J.A. Johannessen, and B. Chapron, (2005), On radar imaging of current features. Part 1: Model and comparison with observations, Journal of Geophysical Research, Vol. 110, C07017.

Longuet-Higgins, M.S., (1957). The statistical analysis of a random moving surface, Phil. Trans. Roy. Soc. London, Ser.A 249, 321-387

Mouche, A.A., D. Hauser and V. Kudryavtsev (2006), Radar scattering of the ocean surface and sea-roughness properties: A combined analysis from dual-polarizations airborne radar observations and models in C-band. Journal of Geophysical Research, 111, C09004, doi:10.1029/2005JC003166

Mouche, A.A., B. Chapron and N. Reul (2007a), A Simplified Asymptotic Theory for Ocean Surface Electromagnetic Waves Scattering, Waves in Random and Complex Media, Volume 17, Issue 3 August 2007 , pages 321 - 341. DOI: 10.1080/17455030701230261.

Mouche, A. A., B. Chapron, N. Reul, D. Hauser, Y. Quilfen (2007b), Importance of the sea surface curvature to interpret the normalized radar cross section, J. Geophys. Res., 112, C10002, doi:10.1029/2006JC004010.

Mouche, A. A., B. Chapron, N. Reul, F. Collard, (2008), Predicted Doppler shifts induced by ocean surface wave displacements using asymptotic electromagnetic wave scattering theories. Waves in Random and Complex Media, Vol.18, Issue 1, pp. 185-196, doi:10.1080/17455030701564644.

Phillips, O. M. (1985), Spectral and statistical properties of the equilibrium range in windgenerated gravity waves, Journal of Fluid Mech., 156, pp. 505- 531. 


\section{Figures}
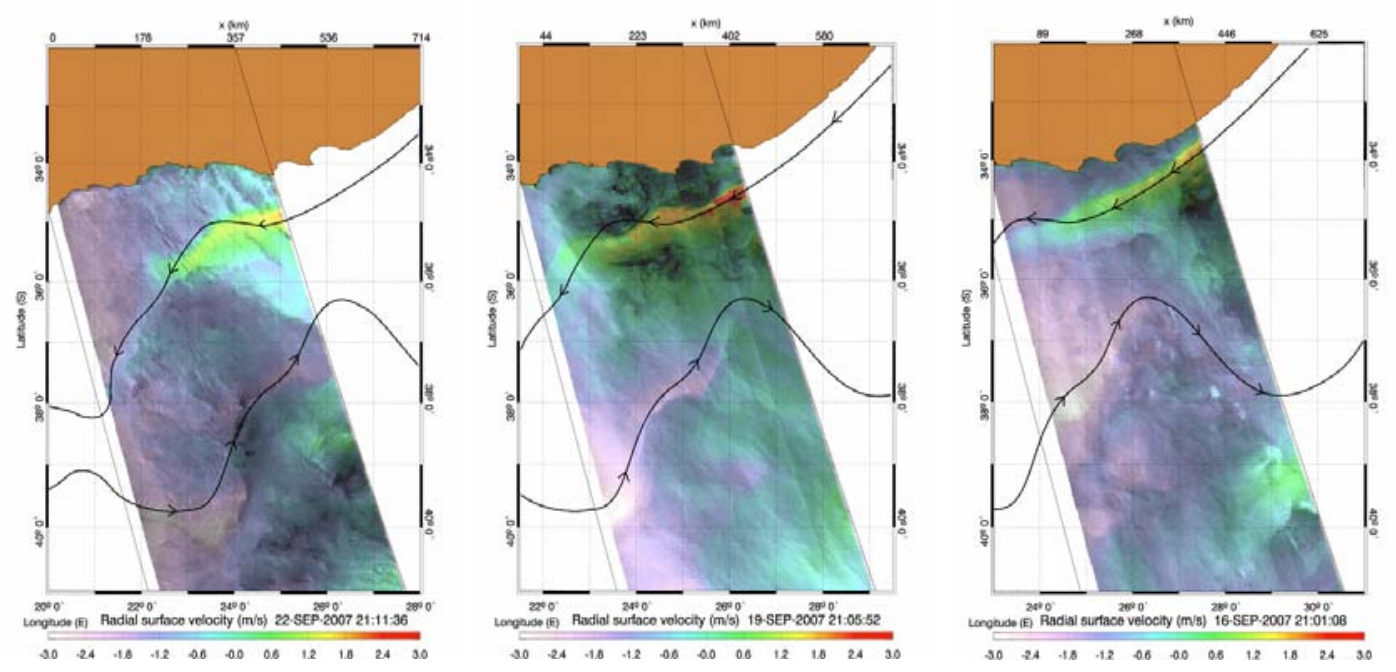

Figure 1

Figure 1. Time series of the Doppler velocity from the ascending ASAR wide swath (420 km) images on 16, 19 and 22 September 2007 (right-to-left) covering the greater Agulhas Current region. The color bar marks the radial velocities from $-3 \mathrm{~m} / \mathrm{s}$ to $+3 \mathrm{~m} / \mathrm{s}$. Positive speed is directed towards the SAR look direction. Black curve marks position of the maximum geostrophic current derived from altimetry 7-day mean. 

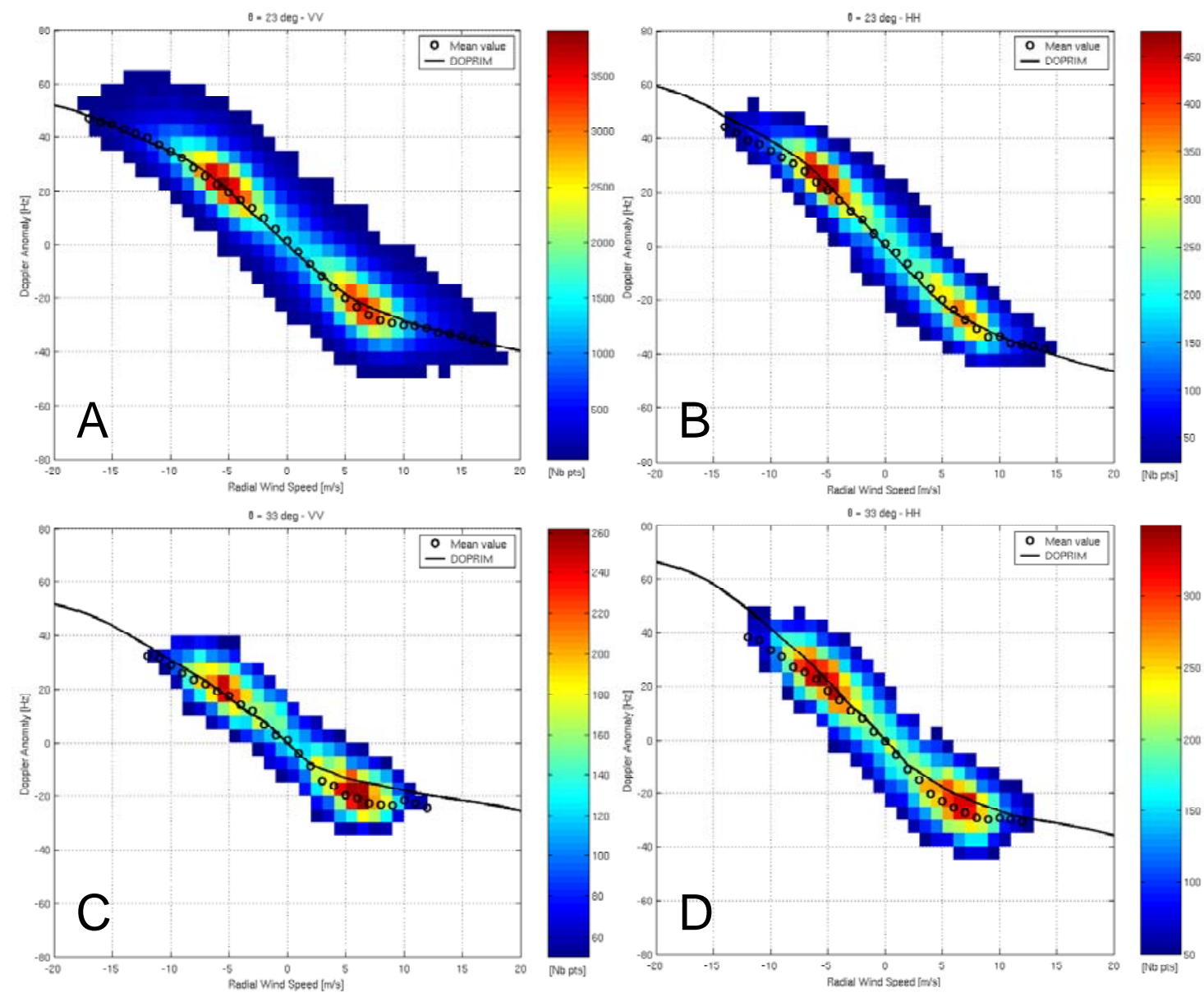

Figure 2

Figure 2. Observed WM (color) and simulated (solid) wind dependence of C-band Doppler shift $\mathrm{VV}$ polarization in (A) and (C) and $\mathrm{HH}$ polarization in (B) and (D) at (top) $23^{\circ}$ and (bottom) $33^{\circ}$ incidence angles. The color represents the spread in number of observation points. The open circles mark the mean fit to the observations. Upwind corresponds to positive radial velocity. 


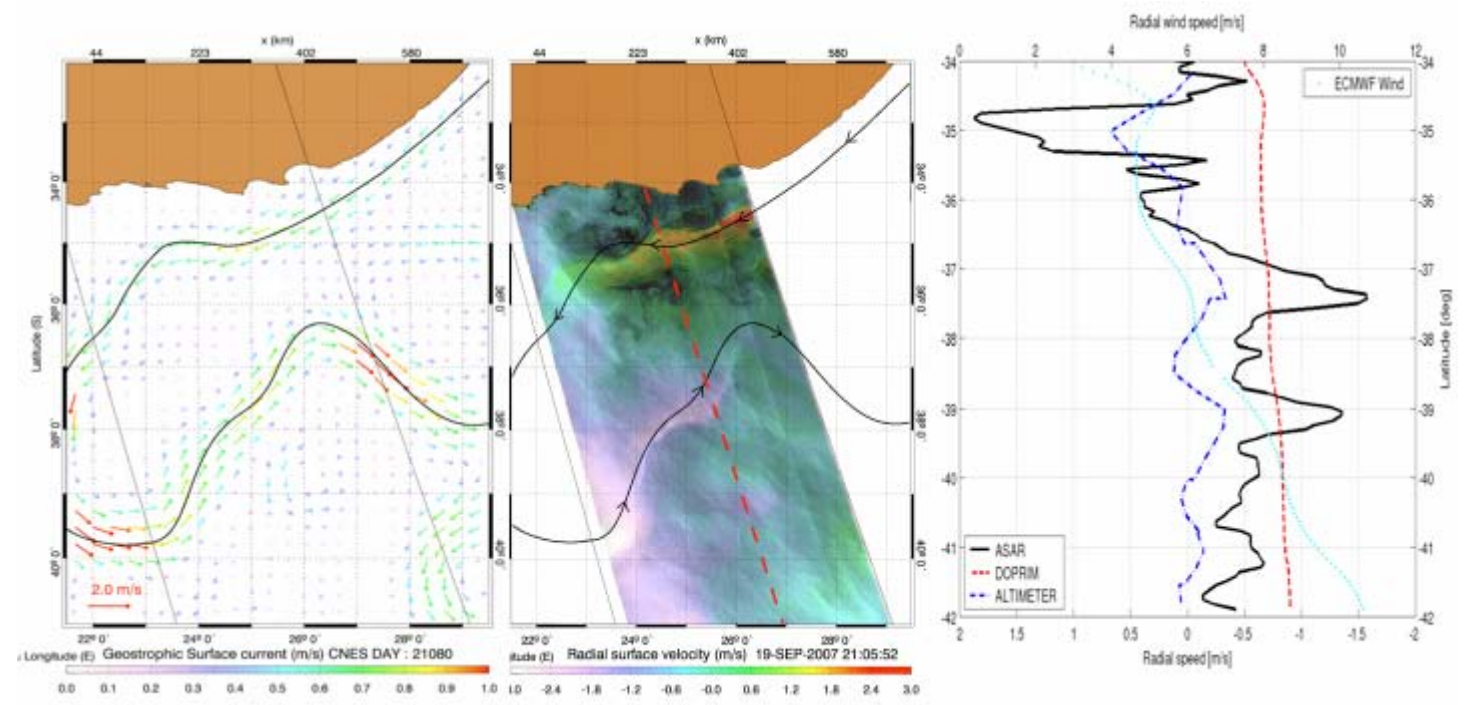

Figure 3

Figure 3. (left) Weekly mean surface geostrophic current map at $25 \mathrm{~km}$ resolution derived from radar altimetry from 15-22 September 2007 with the location of maximum velocities from the 7-day mean superimposed. (middle) ASAR WSM Doppler velocity map from 19 September. (right) Comparison of range directed velocity profiles along the red azimuth oriented transect marked in the Doppler velocity map (middle) of: (i) observed total Doppler velocity (solid black line), (ii) surface geostrophic current component (blue dash-dot), (iii) simulated wind induced Doppler velocity (red stippled) and (iv) ECMWF derived wind speed profile (light-blue dotted line). 